\title{
Research Progress of Logarithmic Crown of Bearing Roller
}

\author{
Guo Yangyang $^{1 *}$, Lei Xianqing ${ }^{1}$ and Wang Yongjun ${ }^{1}$ \\ ${ }^{1}$ School of Mechatronics Engineering, Henan University of Science and Technology, Luoyang, HeNan, 471003, China.
}

\begin{abstract}
In view of the complexity of the problem of the logarithmic crown of the bearing roller, this paper introduce the design, processing and measurement of the logarithmic crown, analyze the research progress in various aspects, and explore the unsolved problems.
\end{abstract}

\section{Introduction}

Bearing is an important basic part of all kinds of mechanical equipment. Bearing is widely used in aerospace, high-speed rail, precision machine tools and other fields. After long-term development, China's bearing industry has a strong technical strength and production capacity.

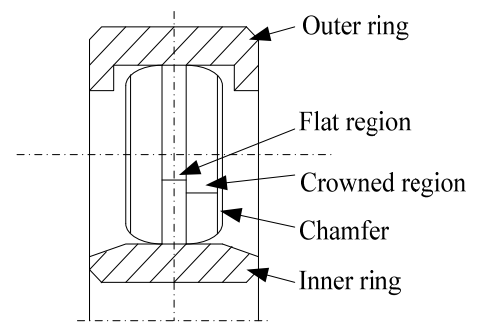

Figure 1. Schematic drawing of a crowned roller in a roller bearing.

In the initial stage, the generatrix form of cylindrical (tapered) roller and raceway is generally designed as a straight line[1]. Due to the serious stress concentration at both ends of the contact between the roller and raceway in the form of straight generatrix, compared with other contact areas, fatigue pitting is more likely to occur, which is the so-called edge effect[2-3]. Based on the research of "edge effect" and stress distribution, Lundberg put forward the basic theory of profile modification of roller bearing bus bar crown[4]. Through fine machining, the roller's plain line has a curve shape slightly protruding from the outside of the body, As shown in figure 1 , so that the contact stress between the roller and the raceway and the lubricating oil film are evenly distributed along the length direction of the contact area[5]. At present, the basic line types of the roller crown prime line used in the project include full crown type, arc correction type, logarithmic curve type, etc[6-7]. It is generally believed that the logarithmic curve has the most obvious advantage, which can not only greatly reduce the "edge effect", but also the stress distribution along the middle of the axis of the roller is relatively uniform, which is of great help to improve the bearing capacity and service life of the bearing.

\section{Design, processing and measurement of logarithmic crown of bearing roller}

\subsection{Design of logarithmic crown of bearing roller}

In crown design, SKF, FAG, NSK and other foreign companies have conducted in-depth research. Luoyang Bearing Research Institute, Zhejiang University and other research institutes have done a lot of research in the design of rolling body crown. Since Lundberg summed up the theoretical logarithmic convex equation, many scholars have made improvements and corrections on this basis[8-11].

There are several representative forms of logarithmic curve crown:

\subsubsection{Lundberg logarithmic curve convex}

$$
T(x)=\frac{Q\left(1-\gamma^{2}\right)}{E a \pi} \ln \frac{1}{1-(x / a)^{2}}
$$

With the following design parameters: $E$ is Young's modulus, $Q$ is load, $a$ is $1 / 2$ effective contact length, $\gamma$ is Poisson's ratio.

\subsubsection{Convex type of Johns Gohar logarithmic curve}

Johns Gohar corrects the defects of Lundberg logarithmic curve (there is still edge stress concentration) and obtains the convex form of Engineering logarithmic curve. The expression is as follows:

$$
T(x)=\frac{Q\left(1-\gamma^{2}\right)}{E a \pi} \ln \frac{1}{1-(1-0.3 b / a)(x / a)^{2}}
$$


With the following design parameters: $E$ is Young's modulus, $Q$ is load, $a$ is $1 / 2$ effective contact length, $b$ is $1 / 2$ effective contact width, $\gamma$ is Poisson's ratio.

\subsubsection{Hiroki Fujiwara, Tatsuo Kawase logarithmic curve convex}

Due to the phenomenon of edge stress caused by the inaccuracy of convex roller of logarithmic curve, Hiroki Fujiwara and Tatsuo Kawase optimized their design, introduced three parameters $s_{1}, S_{2}, Z_{m}$ and obtained a new logarithmic curve formula:

$$
T(x)=\frac{s_{1} Q\left(1-\gamma^{2}\right)}{E a \pi} \ln \frac{1}{1-\left\{1-\exp \left[\frac{Z_{m} \pi a E}{s_{1} Q\left(1-\gamma^{2}\right)}\right]\right\}\left(1+\frac{x-a}{s_{2} a}\right)^{2}}
$$

With the following design parameters: $s_{1}$ is the comprehensive loads; $S_{2}$ is the ratio of crown length to $1 /$ 2 effective contact length; and $Z_{m}$ is the convex measurement of roller end.

\subsection{Processing of logarithmic crown of bearing roller}

The crown of the roller is machined by grinding and superfinishing[12-15]. Grinding is generally used for the machining and manufacturing of roller with low precision or large size, as well as the pre grinding of roller crown. The logarithmic curve profile and crown measurement of grinding roller depend on the profile of grinding wheel and guide wheel working face and the dressing precision. There is no need for special tools, but the difficulty lies in how to accurately simulate the required logarithmic curve profile. The foreign cut in grinding machine can grind the outer diameter surface

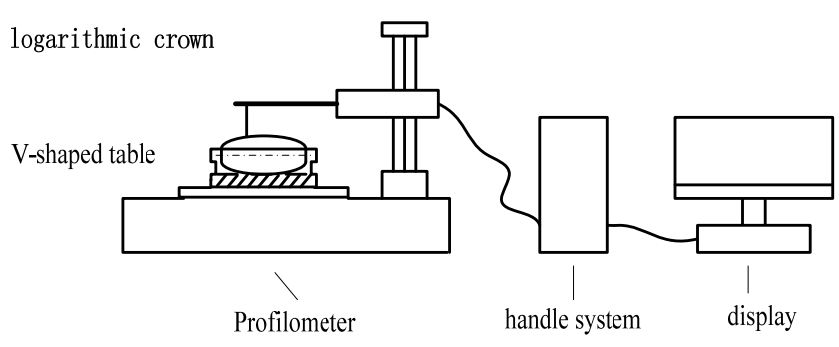

Figure 2. Measuring device.

\section{Conclusions}

There are many researches on bearing crown at home and abroad, but most of them are about bearing crown design and machining in the existing literature. Some equations and crown measures are optimized, and then the simulation is carried out to draw a conclusion, but there is no experimental verification. The main reason for this and the ball base surface at the same time, but the difficulty lies in that the program needs to input more coordinate points to determine the logarithmic curve profile, and a full set of tooling is required when replacing parts; the cost is expensive and the tooling accuracy is required to be high.

At present, in China, the centerless through superfinishing machine is usually used for crown machining, but the guide roller design is not fully mature, which requires many times of trial superfinishing to repair, and the production cycle is restricted; The current research can only show that the super precision grinding can form the crown curve, but it can not show the specific shape and size of the crown curve. The mechanism and law of the grinding are not clear in theory. In the actual production and processing process, the precision grinding of bearing parts has always been in the state of experience leading.

\subsection{Measurement of logarithmic crown of bearing roller}

It is very important to measure whether the profile of the processed roller conforms to the requirements of process design and how much the error value is. The crown shape and value of the roller can be ensured by detecting the crown shape curve and value, adjusting the processing equipment or grinding the guide roller according to the measurement results. Many scholars have done a lot of research and put forward practical methods[16-18].

The domestic research generally focuses on the summary and exchange of the technical practice of the instrument. As shown in figure 2, The measuring instrument basically depends on the imported precision profilometer, and uses the designed linetype to judge whether it is qualified. Because the processing technology can not explain the specific line shape of the processing, it can not be fitted with the known line shape. So it is very important to study the fitting method.

phenomenon is the lack of basic research on fitting problem. Only through careful research can the precise and efficient modification of crown be achieved Processing provides strong theoretical and technical support. 


\section{Acknowledgments}

The authors gratefully acknowledge Teacher's care and guidance.

\section{References}

1. Xu Shuping, Kang Shichen, R.A. (2008)Crown design and measurement method of rolling bus of tapered roller bearing . Journal of Shenyang University of technology, 27 (05): 17-18.

2. Raja Kountanya, Vijay Jagdale, R.A.(2015)Frictionless elastic contact of crowned roller: approximate analytical calculation of compliance and contact area. Journal of Engineering Tribology, 229(10): 1206-1213.

3. Juan X U, Niu Q, Huaming Q I, et al, R.A.(2012)A research on the contact stress of roller bearing based on crowning analysis. Computer Aided Drafting Design and Manufacturing, 22(2):55-58.

4. Lundberg G, R.A.(1939)Elastische beruehrung zweierhalbraeume. Forchung Auf Dem Gebiete Des Ingenieur Wesens , 10(5): 201-211.

5. Palmgren G, J. (1949)Cylinder compressed between two plane bodies. 2nd Sweden: SKF Gotebory. : 65-77.

6. Wang L Q, Ye Z H, Gu L, R.A.(2011) The effect of roller profile modification on roller bearing performance . Advanced Materials Research, 230 /232: $1210-1215$.

7. Vancanh Tong, Seongwook Hong, R.A.(2017)Statistical investigation into the effects of distributed roller diameter error on the fatigue life of tapered roller bearings [J]. Journal of Mechanical Science and Technology.31(12):5977-5985.

8. Johns P M, Gohar R, R.A.(1981)Roller bearings under radial and eccentric loads. Tribology International, 14(3): 131-136.

9. Ma Jiaju, $\mathrm{Xu}$ Wen, Liu shuangbiao, R.A.(1997)engineering design of logarithmic roller. Bearing, (6): 25.

10. Hiroki Fujiwara, Takuji Kobayashi, Tatsuo Kawase, et al, R.A.(2010)Optimized logarithmic roller crowning design of cylindrical roller bearings and its experimental demonstration. Tribology Transactions, 53: 909-916.

11. Hiroki Fujiwara Tatsuo Kawase, R.A.(2006)Logarithmic profiles of rollers in roller bearings and optimization of the profiles. the Proceedings of the Japan Society of Mechanical Engineers Part C. :3022-3029.

12. Gao Zuobin, Ma Wei, Deng Xiaozhong, R.A. ( 2011)Research progress of roller bearing crown machining technology. Bearing, 10: 61-63.

13. Gao Zuobin, R.A.(2004)Analysis of the mechanism of the through lapping of tapered roller. Bearing, 04 : 12-14.

14. $\mathrm{Hu}$ Shigang, R.A. (1997)Study on process adaptability of ultra precision grinding guide roller of logarithmic convex cylindrical roller. Mechanical and electrical engineering, 05: 35-37.

15. Liu Qi, Gao Wuzheng, Wang Mengyin, R.A. (2016)Processing technology and testing method of logarithmic curve profile roller. Bearing, 12: 12-14.

16. Ji Wenchao, Chen Xiaoyang, Lu Jincai, etc, R.A. (2015)comparative measurement of profile of crown roller. Bearing,07: 50-53.

17. Lei Xianqing, Zhang Yadong, Ma wensuo, et al, R.A. (2018)Least square fitting and error evaluation of roller profile of bearing. Optical precision engineering, 08: 2039-2047.

18. Lei Xianqing, Man Ruidong, Gao Zuobin, et al, R.A. (2017)Geometric traversal search and evaluation algorithm for log curve profile error. Mechanical design and manufacturing, 08: 54-56. 